\title{
STATISTICAL PROPERTIES OF RADIO-RICH CORONAL MASS EJECTIONS
}

\author{
N. GOPALSWAMY ${ }^{1,2}$, S. YASHIRO ${ }^{1,2}$, G. MICHALEK ${ }^{1,2}$, M. L. KAISER ${ }^{2}$, R. A. HOWARD ${ }^{3}$, and \\ J.-L. BOUGERET ${ }^{4}$ \\ ${ }^{I}$ Center for Solar Physics and Space Weather, Department of Physics, The Catholic University of America, \\ Washington DC 20064, USA \\ ${ }^{2}$ NASA Goddard Space Flight Center, Greenbelt, MD 20771, USA \\ ${ }^{3}$ Naval Research Laboratory, Washington DC 20375, USA \\ ${ }^{4}$ Paris Observatory, Meudon, France
}

\begin{abstract}
Coronal mass ejections (CMEs) that produce type II radio bursts in the near-Sun interplanetary medium are termed radio-rich owing to their ability to drive MHD shocks. We summarize the statistical properties of these CMEs in order to see if they constitute a special population distinct from the general population. We found that these CMEs are faster and wider than the regular CMEs and show significant deceleration within the coronagraph field of view. Most of these CMEs were also found to be proton accelerators. We conclude that these type II bursts may be indicative of geoeffective CMEs and hence relevant to space weather.
\end{abstract}

\section{INTRODUCTION}

Coronal mass ejections (CMEs) that produce type II radio emission in the Decameter-Hectometric (DH) wavelengths (1-14 MHz) are known as radio-rich CMEs (Gopalswamy et al., 2000a, 2001a). They drive shocks in the outer corona ( $>2 \mathrm{Rs}$ ) that accelerate electrons, which in turn produce type II radio bursts (Reiner and Kaiser, 1999). The DH radio window became available through the WAVES experiment (Bougeret et al., 1995) on board the Wind spacecraft launched in 1994. Since the DH type II bursts are indicative of shocks just departing near the Sun, they may provide an excellent source of advanced warning of geoeffective solar eruptions (Webb et al., 2000). When the shocks are near the Sun, they accelerate solar energetic particles (SEPs), which arrive at 1 AU within tens of minutes (Tylka, 2001; Reames, 1999). The IP shocks arriving at 1 AU signal the sudden commencement of geomagnetic storms. They also bring along a population of locally accelerated SEPs known as energetic storm particles (ESPs), which can be potentially hazardous to astronauts and space-based technological systems. Sometimes, the shock sheath can also cause geomagnetic storms if it contains southward magnetic field component. When the eruption occurs close to the Sun center, an ejecta usually follows the shock (Burlaga et al., 1981, Gopalswamy et al., 2000b). Thus studying the radio-rich CMEs may help us identify the population of geoeffective CMEs.

\section{DATA}

In order to see what is special about the DH CMEs as compared to the regular CMEs, we examined the speed, width and acceleration of the radio-rich CMEs and also their association with major SEP events. The Solar and Heliospheric Observatory (SOHO) mission's Large Angle and Spectrometric Coronagraph (LASCO) detected the CMEs. The WAVES experiment's high frequency radio detector RAD2 provided the dynamic spectra of the type II bursts. From the $\mathrm{C} 2$ and $\mathrm{C} 3$ coronagraph images of LASCO, we measured the height-time variation of CMEs. Linear and quadratic fits to the height-time plot were used to derive the speed and acceleration of the CMEs. We used the online Solar Geophysical Data to obtain the solar sources of the CMEs. The solar sources were confirmed 
by running EUV movies from SOHO's Extreme-ultraviolet Imaging Telescope. Information on the SEPs was also obtained from the Solar Geophysical Data. For the SEPs we simply checked the association with the DH type II bursts. We have provided an illustrative example of the data sets in Figure 1: the 2001 August 15 CME was a backsided event from the southwest quadrant. The CME was first seen above the occulting disk at 23:30 UT and moved with a speed of $1575 \mathrm{~km} / \mathrm{s}$. In radio, the event started with an SA ("shock-associated") event at $2.5 \mathrm{MHz}$, followed by a type II burst at $5 \mathrm{MHz}$. The type II burst from limb events is most likely at twice the local plasma frequency, so the burst must have originated from the corona where the local plasma frequency is $2.5 \mathrm{MHz}$ (density $=7.75 \times 10^{4}$ $\mathrm{cm}^{-3}$ ). The density at the coronal base is much larger (a few times $10^{8} \mathrm{~cm}^{-3}$ ) so the low starting frequency of the type II burst must be due to occultation by the overlying corona.

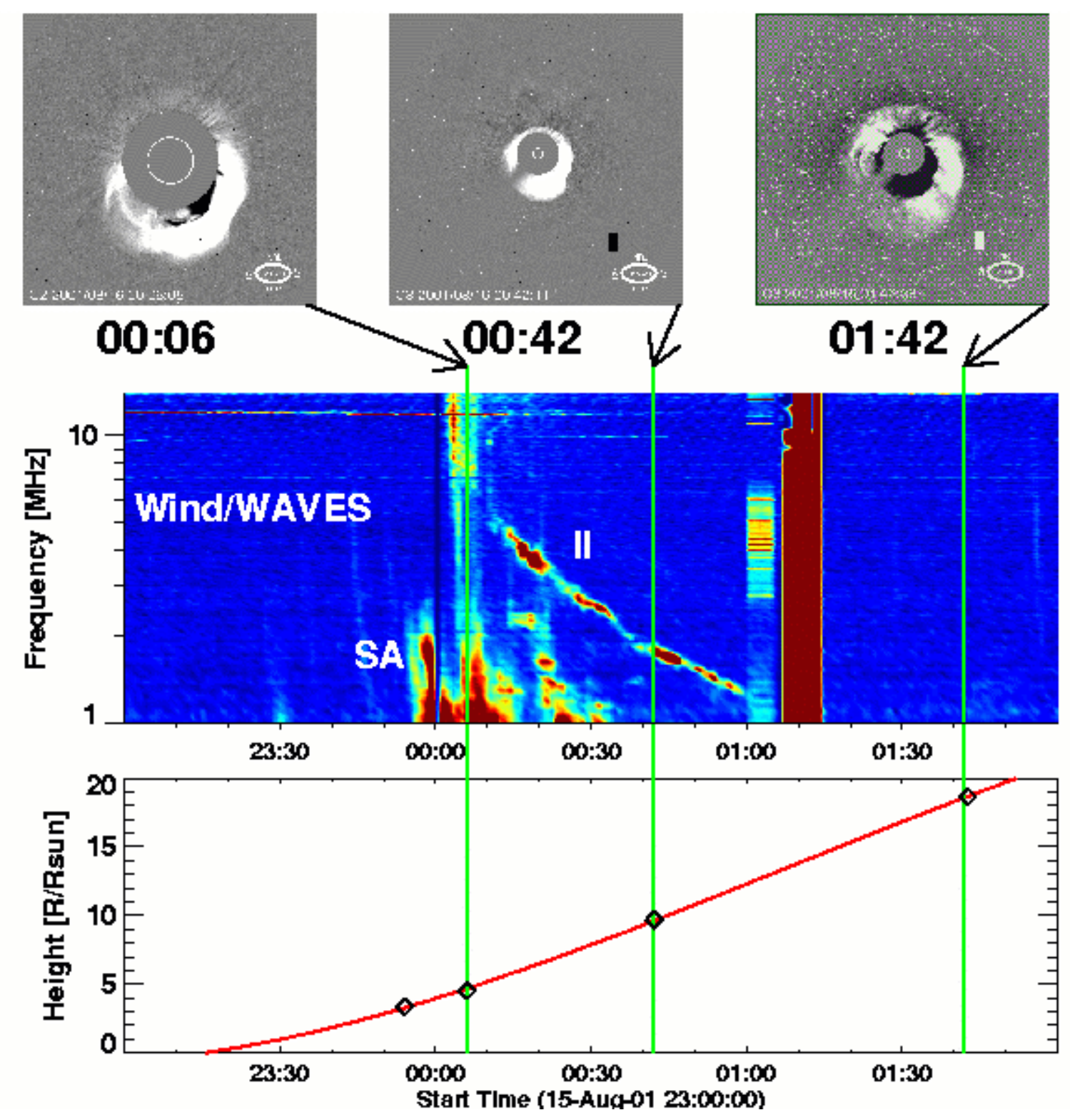

Fig.1 SOHO/LASCO images (top) at three instances (00:06, 00:42, and 01:42 UT) during the DH type II burst (middle) and the CME height-time plot (bottom) for the 2001 August 15 event. The Type II burst and the "shock associated" events are marked as II and SA, respectively. By 01:42 UT, the radio emission has moved to frequencies below $1 \mathrm{MHz}$. The CME had an average speed of $1575 \mathrm{~km} / \mathrm{s}$. The vertical lines mark the times of the three LASCO images. The 01:42 UT LASCO image indicates the arrival of SEPs at the L1 point. The dark disc in LASCO images is the occulting disk and the white circle represents the optical Sun. The rectangular features after 01:00 UT represent calibration interval. 
Figure 1 also shows three LASCO images corresponding to various instances of the type II burst. The outer edge of the CME can be taken as the approximate coronal height from which the type II emission is generated. The type II burst continued to lower frequencies beyond the RAD2 spectral range (not shown). The height-time plot of the CME shown in Figure 1 indicates where the type II burst might originate at later times. The LASCO image at 01:42 is severely streaked with SEPs hitting the SOHO detector. This is image corresponds roughly to 2 hours after the CME onset.

\section{SUMMARY OF RESULTS}

A detailed analysis of the statistical properties of CMEs associated with DH type II bursts was recently published by Gopalswamy et al. (2001a). Here we provide a brief summary of the results extended to a larger set of events during the study period January 1996 to June 2001. In all there were 132 DH type II bursts that had overlapping observations from SOHO. The statistical properties of the CMEs associated with the DH type II bursts are shown in Figure 2. We can infer the following: (1) the average speed of the DH CMEs $(1030 \mathrm{~km} / \mathrm{s})$ is more than two times larger than that of regular CMEs $(\sim 450 \mathrm{~km} / \mathrm{s})$. Only $16 \%$ of the CMEs had speed around the average value of regular CMEs. We have not corrected for the projection effects so the speeds in Figure 1 represent a lower limit (Gopalswamy et al., 2000a). (2) The typical width of regular CMEs is $\sim 60^{\circ}$. This is in sharp contrast to the width distribution of DH CMEs shown in Figure 2 (middle panel). Only 7\% of the CMEs have width in the average range. Another important result is that a majority of the DH CMEs are halo events. It is difficult to know the width of the halo CMEs, but it is significant that 88/132 CMEs were halo events. In other words, 93\% of the DH CMEs are either halo events or have above-average widths. (3) Both positive and negative accelerations are found, but there is a clear bias towards events with deceleration. About $24 \%$ of the events show very little acceleration. Events with deceleration constitute the largest fraction (45\%). The remaining 31\% show acceleration. The measured acceleration of the CMEs within the coronagraph field of view depends on the relative contribution from the propelling and retarding forces. We infer that the coronal drag may play a significant role in deciding the propagation of CMEs in the IP medium.
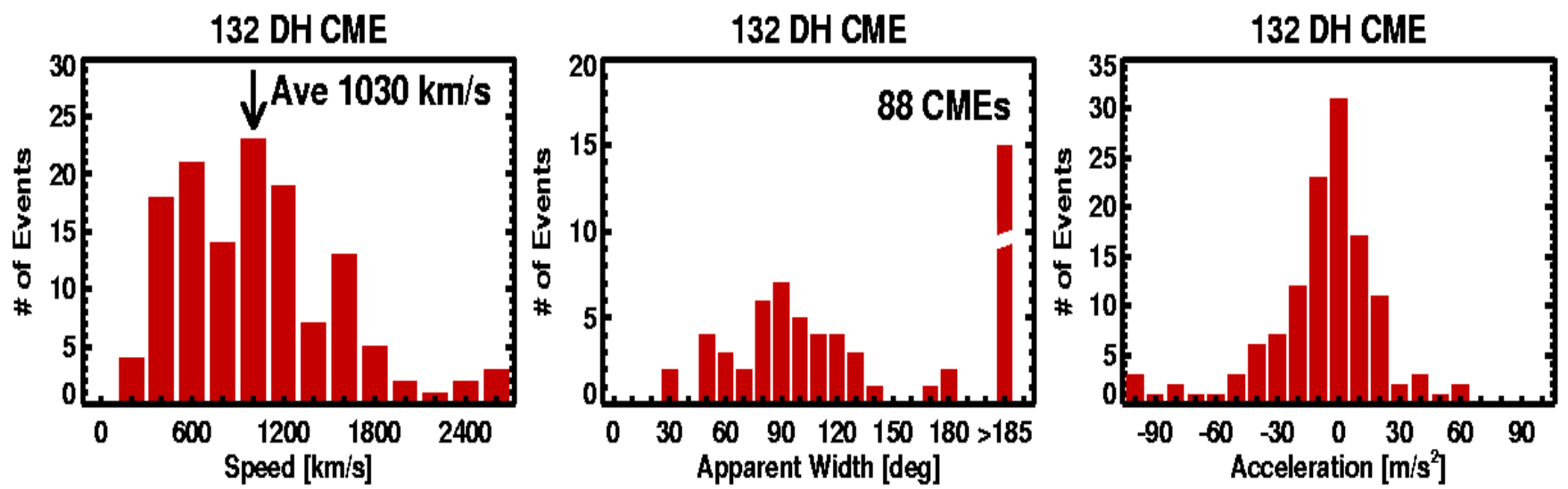

Fig. 2: Histograms of speed (left), width (middle) and acceleration (right) of the 132 radio-rich CMEs considered in this study. The average speed of the distribution is marked. The last bin in the width histogram includes all CMEs with a measured width greater than $185^{\circ}$. This bin is shown broken because it was out of scale with 88 CMEs.

We also examined the association between the major SEP events of this solar cycle until October 2001. There were 44 proton events that could be classified as major SEP events. All but three of the SEP events had DH type II bursts. In the three events without DH type II bursts, there were clear "shock associated" type III events starting at frequencies below $2 \mathrm{MHz}$. These events usually accompany type II bursts. We also did not examine the possibility of type II bursts starting at frequencies below $1 \mathrm{MHz}$. The 
preliminary investigation suggests that the electron accelerators are also good proton accelerators. When we examined the fast CMEs without associated DH type II bursts, we found them to be of average width. We need to examine additional factors such as ambient Alfven speed before making a firm conclusion as to why these fast CMEs were not associated with DH type II bursts.

\section{DISCUSSION AND CONCLUSIONS}

The primary result of this study is that DH type II bursts are indicative of faster and wider CMEs. Though the solar sources of these DH CMEs were from all longitudes, most of them were halo or partial halo CMEs. This may prove to be a useful way to identify geoeffective CMEs, even if white light observations are not available. The CME width also seems to be a critical parameter in deciding whether a fast CME is radio-rich or not. The large apparent width of the DH CMEs suggest the possibility that most of these CMEs may expand rapidly in all directions, in addition to the rapid outward motion. Gopalswamy et al. (2001b) pointed out such a possibility from independent considerations. The observed deceleration of DH CMEs within 30 solar radii from the Sun may indicate that the coronal drag is an important factor that determines the propagation characteristics of fast CMEs in the interplanetary medium. The connection between SEPs and DH type II bursts is being investigated, but the preliminary results indicate that the west-limb DH type II bursts are also good indicators of SEP accelerators.

\section{ACKNOWLEDGMENT}

This work was supported by NASA, Air Force Office of Scientific Research, and NSF.

\section{REFERENCES}

Bougeret, J.-L., M. L. Kaiser, P. J. Kellogg, R. Manning, K. Goetz, S. J. Monson, N. Monge, L. Friel, C. A. Meetre, C. Perche, L. Sitruk, and S. Hoang, Waves: The Radio and Plasma Wave Investigation on the Wind Spacecraft, Space Sci. Rev., 71, 231, 1995.

Burlaga, L., Sittler, E., Mariani, F., and Schwenn, R., Magnetic loop behind an interplanetary shock - Voyager, Helios, and IMP 8 observations, J. Geophys. Res., 86, 6673, 1981.

Gopalswamy, N., Y. Hanaoka, T. Kosugi, R. P. Lepping, J. T. Steinberg, S. Plunkett, R. A. Howard, B. J. Thompson, J. Gurman, G. Ho, N. Nitta, and H. S. Hudson, On the relationship between coronal mass ejections and magnetic clouds, Geophys. Res. Lett., 25, 2485, 1998.

Gopalswamy, N., M. L. Kaiser, B. J. Thompson, L. F. Burlaga, A. Szabo, A. Vourlidas, A. Lara, S. Yashiro, and J. L. Bougeret, Radio-rich Solar Eruptive Events, Geophys. Res. Lett., 27, 1427, 2000a.

Gopalswamy, N., A. Lara, R. P. Lepping, M. L. Kaiser, D. Berdichevsky, and O. C. St. Cyr, Interplanetary acceleration of coronal mass ejections, Geophys. Res. Lett., 27, 145, $2000 \mathrm{~b}$.

Gopalswamy, N., S. Yashiro, M. L. Kaiser, R. A. Howard, and J.-L. Bougeret, Characteristics of coronal mass ejections associated with long-wavelength type II radio bursts, J. Geophys. Res., 106 , 29,219, 2001a.

Gopalswamy, N., A. Lara, S. Yashiro, M. L. Kaiser, and R. A. Howard, Predicting the 1-AU arrival times of coronal mass ejections, J. Geophys. Res., 106, 29,207, $2001 \mathrm{~b}$.

Reames, D. V., Particle acceleration at the Sun and in the heliosphere, Space Sci. Rev., 90, 413, 1999.

Reiner, M. J. and M. L. Kaiser, High-frequency type II radio emissions associated with shocks driven by coronal mass ejections, J. Geophys. Res, 104, 16,979, 1999.

Tylka, A., New insights on solar energetic particles from Wind and ACE, J. Geophys. Res., 106, 25,233, 2001.

Webb, D. F., E. W. Cliver, N. U. Crooker, O. C. St. Cyr, and B. J. Thompson, J. Geophys. Res., 105, 7491, 2000. 